\title{
Dissolved organic carbon and nitrogen dynamics in the Douro River estuary, Portugal
}

\section{Dinámica del carbono y nitrógeno orgánico disueltos en el estuario del Duero, Portugal}

\author{
C Magalhães ${ }^{1 *}$, C Teixeira ${ }^{1,2}$, R Teixeira ${ }^{1}$, A Machado $^{1}$, I Azevedo ${ }^{1,2}$, AA Bordalo ${ }^{1,2}$ \\ ${ }^{1}$ Centre of Marine and Environmental Research (CIIMAR), University of Porto, Porto, Portugal. \\ *E-mail: c_magalhaes@yahoo.com,cmag@icbas.up.pt \\ 2 Laboratory of Hydrobiology, Institute of Biomedical Sciences (ICBAS-UP), University of Porto, Porto, Portugal.
}

\begin{abstract}
Dissolved organic carbon (DOC) and dissolved organic nitrogen (DON) are metabolically important in estuaries. Their availability is influenced by organic matter inputs (internal and external) as well as by internal microbial transformations within the system. In this study, spatial and seasonal dynamics of DOC and DON were evaluated in the Douro River estuary (Portugal). Monthly surveys were performed from October 2005 to December 2006 along a salinity gradient at three different depths. DOC and DON were calculated by subtracting the respective inorganic components (dissolved inorganic carbon, DIC, and dissolved inorganic nitrogen, DIN) from total dissolved carbon (TDC) and total dissolved nitrogen (TDN). In the Douro River estuary, DIC exhibits a linear and positive distribution as a function of salinity, indicating an input of DIC into the estuary from coastal waters. This pattern of distribution along the salinity gradient was also observed for total particulate matter, since a decreasing trend upstream was observed. In contrast, the results showed a general nonconservative behaviour of DOC along the estuarine transect during most months. This typical deviation from conservative mixing was mainly associated with sewage DOC sources into the lower and middle estuary. On average, DON concentrations represented 52-88\% of the TDN. Along the transect, relationships between DIN and DON with salinity were generally conservative, decreasing towards the mouth, highlighting the fluvial source of such nitrogen forms; however, occasional DON sewage loads were registered. Estimated global Douro River watershed exports of DOC and DON to coastal waters yielded values of $420 \mathrm{~kg} \mathrm{C} \mathrm{m}^{-2} \mathrm{yr}^{-1}$ and $125 \mathrm{~kg} \mathrm{~N} \mathrm{~m}^{-2} \mathrm{yr}^{-1}$, respectively, which are high when compared with previously predicted levels from global models of DOC and DON export.
\end{abstract}

Key words: dissolved organic nitrogen, dissolved organic carbon, Douro River estuary.

\section{Resumen}

El carbono orgánico disuelto (COD) y el nitrógeno orgánico disuelto (NOD) son compuestos metabólicamente importantes en los sistemas estuarinos. Su disponibilidad es influenciada por aportes de materia orgánica (internos y externos) y por transformaciones microbianas internas. En este trabajo se evaluaron las dinámicas espaciales y temporales del COD y NOD en el estuario del Río Duero. Los muestreos se realizaron mensualmente entre octubre de 2005 y diciembre de 2006 a lo largo del gradiente salino del estuario, a tres profundidades distintas. El COD y NOD fueron calculados mediante la substracción de los respectivos componentes inorgánicos del CDT (carbono disuelto total) y el NDT (nitrógeno disuelto total). El carbono inorgánico disuelto (CID) en el estuario del Duero mantiene una relación lineal positiva con la salinidad, indicando un aporte de CID al estuario por parte de las aguas costeras. Este patrón de distribución a lo largo del gradiente salino fue también observado para el material particulado total, ya que se observó una tendencia decreciente corriente arriba. En contraste, los resultados mostraron un comportamiento no conservativo del COD durante gran parte de los meses, en su mayoría asociado al aporte de COD de origen antropogénico en las porciones media y inferior del estuario. Las concentraciones de NOD representan 52-88\% del NDT. A lo largo del transecto las relaciones entre nitrógeno inorgánico disuelto (NID) y NOD con la salinidad mostraron un comportamiento conservativo, lo que indica una fuerte influencia del Río Duero en las concentraciones de NID y NOD del estuario. No obstante, se han registrado aportes ocasionales de NOD de origen antropogénico. Las exportaciones globales de COD y NOD de la cuenca hidrográfica del Duero a las aguas costeras fueron estimadas en $420 \mathrm{~kg} \mathrm{C} \mathrm{m}^{-2}$ año-1 y $125 \mathrm{~kg} \mathrm{~N} \mathrm{~m}^{-2}$ año ${ }^{-1}$, respectivamente, las cuales son elevadas al compararse con los resultados de modelos de exportacion global de COD y NOD para la misma cuenca.

Palabras clave: carbono orgánico disuelto, estuario del Duero, nitrógenio orgánico disuelto.

\section{Introduction}

Dissolved organic matter (DOM) is a major component of the organic matter transported to the coastal zone by rivers (Meybeck 1982, Ludwig et al. 1996). It influences aquatic

\section{Introducción}

La materia orgánica disuelta (MOD) es un componente principal de la materia orgánica transportada a la zona costera por los ríos (Meybeck 1982, Ludwig et al. 1996); influye en las 
food webs, controls the availability of dissolved nutrients and metals, and affects the optical properties of aquatic systems (Findlay and Sinsabaugh 2003). In addition to controlling ecosystem-level processes, DOM is also important from a global and regional biogeochemical perspective, since it constitutes an important pathway for carbon (C) and nitrogen (N) transport from land to sea. These compounds are metabolically important in estuaries and their availability is influenced by organic matter inputs (internal and external) and microbial transformations within the estuaries. The main in situ production mechanisms of DOM include exudation by phytoplankton, egesting by proto- and metazoan grazers, and viral lysis of phytoplankton and bacterial cells (Nagata 2000). The potential for utilization or remineralization of organic growth substrates is determined primarily by the substrate C:N ratio (Goldman et al. 1987). Another important factor affecting DOM dynamics within an estuarine system is human pressure, for example, through the construction of dams, since they change the timing and quantity of freshwater, sediment, inorganic, and organic matters delivered to estuaries and adjacent coastal zones (Hopkinson and Vallino 1995). The discharge of treated or untreated wastewater into estuaries also affects metabolism by increasing allochthonous nutrient or organic matter inputs, respectively. In addition, the importance of hydrology in retaining these elements has been highlighted in several estuarine ecosystems (Nixon et al. 1996).

To better understand these processes, data on dissolved organic nitrogen (DON) and carbon (DOC) are required, which have not been previously recorded on a routine basis in the Douro River estuary. The Douro River originates the largest watershed in the Iberian Peninsula. Its $98,000 \mathrm{~km}^{2}$ are unequally shared between Portugal (20\%) and Spain (80\%). Over 50 large dams have been constructed, especially in the last 50 years, for irrigation and electric power generation purposes, resulting in flow regulation. The estuary is limited upstream by the last dam, located $21.6 \mathrm{~km}$ from the mouth. This dam determines the freshwater flow into the estuary (Vieira and Bordalo 2000) and represents the main source of phytoplankton biomass into the estuary (Bordalo and Vieira 2005). River flow presents large inter-annual variability, with considerable differences between wet and dry years. A decrease in annual flow has been reported due to climate change, but also due to increased water storage for hydroelectric power generation, agriculture abstraction, and domestic consumption (Bordalo and Vieira 2005). The Douro estuary is located in one of the most populated zones of Portugal. Three main cities (Porto, Vila-Nova-de-Gaia, and Gondomar; 700,000 inhabitants) are located within the estuary, exerting an important anthropogenic pressure in terms of urban runoff, sewage discharge, land reclamation, and onshore constructions. A total of eight wastewater treatment plants drain into the estuary. In this article, we focus on studying the sources and distribution of DOC and DON along the salinity gradient of the Douro River estuary. tramas alimenticias, controla la disponibilidad de nutrientes y metales disueltos, y afecta las propiedades ópticas de los sistemas acuáticos (Findlay y Sinsabaugh 2003). Además de controlar procesos a nivel ecosistema, la MOD también es importante desde una perspectiva biogeoquímca regional y global, ya que constituye una importante ruta para el transporte de carbono (C) y nitrógeno $(\mathrm{N})$ de la tierra al mar. Estos componentes son metabólicamente importantes en los estuarios y su disponibilidad está influenciada por los aportes de materia orgánica (internos y externos) y las transformaciones microbianas que allí ocurren. Los principales mecanismos de producción de MOD in situ incluyen su exudado por el fitoplancton, su excreción por organismos proto y metazoarios pastoreadores, y la degradación viral de las células de fitoplancton y bacterias (Nagata 2000). La potencial utilización o remineralización de los sustratos de origen orgánico está determinada primordialmente por la razón C:N (Goldman et al. 1987). Otro factor importante que afecta la dinámica de la MOD en los sistemas estuarinos es la presión antropogénica mediante, por ejemplo, la construcción de presas que modifican las cantidades de agua dulce, sedimento y materia orgánica e inorgánica, que llegan a los estuarios y sus zonas costeras adyacentes, así como el momento en que lo hacen (Hopkinson y Vallino 1995). La descarga de aguas negras, tratadas o sin tratar, a los estuarios también afecta su metabolismo al incrementar los nutrientes alóctonos, o bien, los aportes de materia orgánica, respectivamente. Además, en varios ecosistemas estuarinos se ha evidenciado la importancia de la hidrología para retener estos elementos (Nixon et al. 1996).

Para comprender mejor estos procesos se requieren datos sobre nitrógeno orgánico disuelto (NOD) y carbono orgánico disuelto (COD), los cuales no han sido registrados previa ni rutinariamente en el estuario del Río Duero. Este río drena la principal cuenca hidrológica de la Península Ibérica, cuyos $98,000 \mathrm{~km}^{2}$ son compartidos en diferente proporción entre Portugal (20\%) y España (80\%). Más de 50 grandes presas han sido construidas para irrigación y generación de energía eléctrica sobre su cauce, principalmente en los últimos 50 años, dando como resultado la regulación de su caudal. El estuario del Duero se encuentra limitado hacia el río por la última de estas presas, ubicada a $21.6 \mathrm{~km}$ de la boca. Ésta determina el flujo de agua dulce hacia el estuario (Vieira y Bordalo 2000) y representa la mayor fuente de biomasa fitoplanctónica para el mismo (Bordalo y Vieira 2005). El flujo del río presenta una gran variabilidad interanual, con diferencias considerables entre años lluviosos y secos. Se ha reportado una disminución en el flujo anual debida al cambio climático global, pero también debida al mayor almacenamiento de agua para la generación de energía hidroeléctrica, la agricultura y el consumo doméstico (Bordalo y Vieira 2005). El estuario del Duero se encuentra en una de las zonas más pobladas de Portugal, en cuyas inmediaciones se encuentran tres importantes ciudades (Porto, Vila-Nova-de-Gaia y Gondomar), con un total de 700000 habitantes que ejercen una importante presión 


\section{Methods}

\section{Study area and sampling}

The Douro River estuary has a mesotidal, semi-diurnal regime, an average depth of $8.2 \mathrm{~m}$, and a mean tidal amplitude of $2.8 \mathrm{~m}$. Residence time can reach 14 days during the summer low-flow regime, whereas during high discharge events, the estuary is flushed completely during one tidal cycle and seawater intrusion is prevented during the flood (Vieira and Bordalo 2000). The estuary is vertically stratified even under low river flow conditions (Vieira and Bordalo 2000). Water column temperatures range from $7^{\circ} \mathrm{C}$ in winter to $22^{\circ} \mathrm{C}$ in summer, and salinity in the lower estuary ranges from 0 to 35 .

The data presented in this study were obtained within the framework of a larger sampling program under a contract with the Oporto Water Authority, designed to evaluate the level of fecal contamination of the estuarine water. From October 2005 to December 2006, eight stations were visited monthly along the estuary salinity gradient during ebb tide covering contrasting river discharges (fig. 1). Vertical profiles of temperature, conductivity, salinity, dissolved oxygen, $\mathrm{pH}$, and turbidity were performed with a CTD probe (YSI 6600). Simultaneously, water samples were collected at three depths (surface, middle, and near bottom) with a Van Dorn bottle for the assessment of nutrients (nitrate, nitrite, ammonium), total particulate matter (TPM), particulate organic matter (POM), total dissolved carbon (TDC), total dissolved nitrogen (TDN), and fecal bacteria. Water samples were stored in acid-washed flasks, refrigerated in ice chests, and transported in the dark to the laboratory for processing, no later than $1 \mathrm{~h}$ after collection.

\section{Fecal coliform analysis}

The quantitative determination of fecal coliforms in estuarine water samples was assayed according to the ISO 9308/1

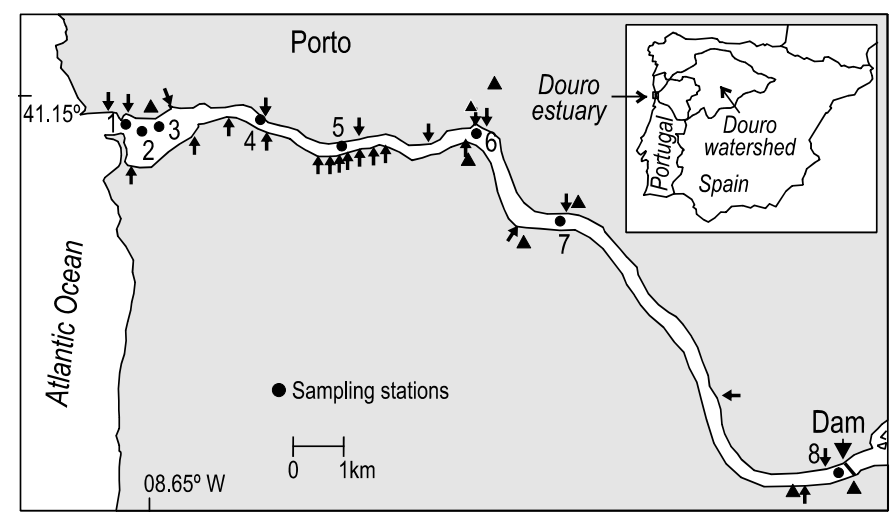

Figure 1. Location of sampling sites $(\bullet)$, wastewater treatment plants $(\boldsymbol{\Delta})$, and tributaries $(\uparrow)$ discharging into the Douro River estuary (Portugal).

Figura 1. Ubicación de sitios de muestreo $(\bullet)$, plantas de tratamiento de aguas negras $(\boldsymbol{\Delta})$, y tributarios $(\uparrow)$ que descargan en el estuario del Duero (Portugal) antropogénica sobre el estuario en términos de drenaje urbano $\mathrm{y}$ aguas negras, terrenos ganados al estuario y construcciones costeras. En total ocho plantas de tratamiento de aguas negras descargan al estuario. En este trabajo se investigaron las fuentes y la distribución del COD y el NOD a lo largo del gradiente salino del estuario del Duero.

\section{Métodos}

\section{Area de estudio y muestreos}

El estuario del Duero tiene un régimen mesomareal semidiurno, con una profundidad media de $8.2 \mathrm{~m}$ y una amplitud mareal media de $2.8 \mathrm{~m}$. El tiempo de residencia del agua puede llegar hasta 14 días durante las mareas muertas de verano, mientras que durante eventos de altas descargas, el estuario se vacía completamente en un solo ciclo de mareas evitando la intrusión del agua de mar durante el flujo (Vieira y Bordalo 2000). El estuario se encuentra verticalmente estratificado aún en condiciones de bajo flujo del río (Vieira y Bordalo 2000). Las temperaturas de la columna de agua fluctuan entre $7^{\circ} \mathrm{C}$ en invierno y $22^{\circ} \mathrm{C}$ en verano, y la salinidad en la parte baja del estuario varía entre 0 y 35.

Los datos presentados en este estudio se obtuvieron en el marco de un programa más amplio de muestreos realizado por contrato con Serviços Municipalizados de Águas e Saneamento do Porto, diseñado para evaluar el nivel de contaminación fecal de las aguas del estuario. Entre octubre de 2005 y diciembre de 2006 se visitaron mensualmente ocho estaciones a lo largo del gradiente salino del estuario, durante la bajamar, cubriendo descargas fluviales contrastantes (fig. 1). Se obtuvieron perfiles verticales de temperatura, conductividad, salinidad, oxígeno disuelto, $\mathrm{pH}$ y turbidez, mediante un CTD YSI 6600. Simultáneamente se recolectaron muestras de agua a tres profundidades (superficie, media agua y fondo) con una botella Van Dorn para evaluar nutrientes (nitrato, nitrito, amonio), materia particulada total (MPT), materia orgánica particulada (MOP), carbono disuelto total (CDT), nitrógeno disuelto total (NDT), y bacterias fecales. Estas muestras fueron almacenadas en frascos lavados con ácido, refrigeradas en hieleras y transportadas en oscuridad al laboratorio para ser procesadas menos de una hora después de su recolección.

\section{Análisis de coliformes fecales}

La determinación de coliformes fecales en las muestras de agua estuarina se realizó de acuerdo con el estándar ISO 9308/ 1 (ISO 1990). Las bacterias se concentraron en filtros estériles de membrana de $0.45 \mu \mathrm{m}$, los cuales fueron colocados en cajas de Petri con medio de cultivo mFC (Merck 10426) e incubados a $44.5^{\circ} \mathrm{C}$. Después de incubarlas por $24 \mathrm{~h}$ se contaron las colonias de coliformes fecales y los datos se expresaron como unidades formadoras de colonias (UFC) por $100 \mathrm{~mL}$ de muestra de agua estuarina. 
standard (ISO 1990). Bacteria were concentrated onto $0.45-\mu \mathrm{m}$ sterile membrane filters, which were placed in Petri dishes containing mFC culture media (Merck 10426) and incubated at $44.5^{\circ} \mathrm{C}$. Fecal coliform bacteria colonies were counted after $24 \mathrm{~h}$ of incubation period and data were expressed as colony-forming units (CFU) per $100 \mathrm{~mL}$ of estuarine water sample.

\section{Analytical procedures}

For TPM and POM assessment, estuarine water samples were prefiltered in precombusted $\mathrm{GF} / \mathrm{F}$ glass-fiber filters (Whatman), and filters dried at $105^{\circ} \mathrm{C}$ and then incinerated at $500^{\circ} \mathrm{C}$ (APHA 1992). Determination of TDC, dissolved inorganic carbon (DIC), and TDN was performed using a Shimadzu Instruments TOC-VCSN analyzer coupled to a total nitrogen measuring unit (TNM-1, Shimadzu). TDC was measured by high temperature catalytic oxidation followed by nondispersive infrared detection of $\mathrm{CO}_{2}$. For DIC determination, samples were automatically acidified $(1.5 \%$ HCL $2 \mathrm{M}$ ) and sparged with carrier gas (purified air) to convert only the inorganic carbon to $\mathrm{CO}_{2}$. DOC was determined by the difference between TDC and DIC. TDN was thermally decomposed in a combustion tube and the resulting nitric oxide detected by chemiluminescence. Three to five replicates were performed for TDC, DIC, and TDN, achieving coefficient variances under $2 \%$. Standard curves of high-grade potassium hydrogen phthalate $\left(\mathrm{C}_{8} \mathrm{H}_{5} \mathrm{KO}_{4}\right)$ and sodium hydrogen carbonate $\left(\mathrm{NaHCO}_{3}\right)$ plus sodium carbonate $\left(\mathrm{Na}_{2} \mathrm{CO}_{3}\right)$ were generated at the beginning of each analytical run for TDC and DIC, respectively. Additional standard curves of high-grade potassium nitrate $\left(\mathrm{KNO}_{3}\right)$ were generated for TDN. The detection limits for TDC, DIC, and TDN were $50 \mu \mathrm{g}$ $\mathrm{L}^{-1}, 4 \mu \mathrm{g} \mathrm{L}^{-1}$, and $0.1 \mathrm{mg} \mathrm{L}^{-1}$, respectively. DON was calculated as the difference between TDN and dissolved inorganic nitrogen (DIN) $\left(\mathrm{NH}_{4}+\mathrm{NO}_{\times}\right)$. Nitrite and ammonium were analyzed following the methods described in Grasshoff et al. (1983). Nitrate was quantified by an adaptation of the spongy cadmium reduction technique (Jones 1984), subtracting nitrite value from the total. All inorganic nitrogen analyses were performed in triplicate and the coefficient of variance was in the range of $0.1 \%$ to $8 \%$ depending on the particular nutrient concentration.

\section{Data analysis}

Statistical analysis was performed at the 95\% confidence level $(P<0.05)$. Data were tested for normality using the Kolmogorov-Smirnov test and for homoscedasticity using Levene's test (Zar 1996). Linear regression analysis (Zar 1996) was conducted to establish relationships between DOC and DIC and DON and DIN, and other environmental parameters measured.

\section{Procedimientos analíticos}

Para la evaluación de la MPT y la MOP se prefiltraron las muestras de agua del estuario a través de filtros de fibra de vidrio GF/F pre-combustionados (Whatman), y filtros secados a $105^{\circ} \mathrm{C}$ y luego incinerados a $500^{\circ} \mathrm{C}$ (APHA 1992). Las determinaciones de CDT, carbono inorgánico disuelto (CID) y NDT se realizaron mediante un analizador Shimadzu Instruments TOC-VCSN acoplado a una unidad medidora de nitrógeno total TNM-1, Shimadzu. El CDT se midió por oxidación catalítica a alta temperatura seguida por la detección infrarroja no dispersiva del $\mathrm{CO}_{2}$. Para determinar el CID se acidificaron de manera automática las muestras con HCL $2 \mathrm{M}$ al $1.5 \%$ y se rociaron con gas transportador (aire purificado) para sólo convertir el carbono inorgánico a $\mathrm{CO}_{2}$. El COD se determinó mediante la diferencia entre CDT y CID. El NDT se descompuso térmicamente en un tubo de combustión, y el óxido nítrico resultante fue detectado mediante quimioluminiscencia. Se analizaron de tres a cinco replicas de CDT, CID y NDT, logrando varianzas del coeficiente menores a $2 \%$. Al principio de cada corrida analítica para CDT y CID se generaron curvas estándar de ftalato ácido de potasio $\left(\mathrm{C}_{8} \mathrm{H}_{5} \mathrm{KO}_{4}\right)$ de alto grado y carbonato ácido de sodio $\left(\mathrm{NaHCO}_{3}\right)$ más carbonato de sodio $\left(\mathrm{Na}_{2} \mathrm{CO}_{3}\right)$, respectivamente. Para el NDT se generaron curvas adicionales de nitrato de potasio $\left(\mathrm{KNO}_{3}\right)$ de alto grado. Los límites de detección para CDT, CID y NDT fueron, respectivamente, $50 \mu \mathrm{g} \mathrm{L}^{-1}, 4 \mu \mathrm{g} \mathrm{L}^{-1}$ and $0.1 \mathrm{mg} \mathrm{L}^{-1}$. El NOD se calculó como la diferencia entre NDT y nitrógeno inorgánico disuelto (NID: $\mathrm{NH}_{4}+\mathrm{NO}_{\times}$). Nitrito y amonio fueron analizados siguiendo los métodos descritos por Grasshoff et al. (1983), mientras que el nitrato se cuantificó mediante una adaptación de la técnica de reducción por cadmio esponjoso (Jones 1984), substrayendo el valor del nitrito del total. Todos los análisis del nitrógeno inorgánico se realizaron por triplicado y el coeficiente de variación estuvo en el rango de $0.1 \%$ a $8 \%$ dependiendo de la concentración del nutriente en particular.

\section{Análisis de los datos}

El análisis estadístico se realizó a un nivel de confianza del $95 \%(P<0.05)$. Se probó la normalidad de los datos usando la prueba de Kolmogorov-Smirnov y su homocedasticidad con la de Leven (Zar 1996). Se realizó un análisis de regresión lineal (Zar 1996) para establecer relaciones del COD y el CID con el nitrógeno (NOD y NID) y otros parámetros ambientales medidos.

\section{Resultados}

La descarga media mensual del río durante el periodo de estudio (octubre de 2005 a diciembre de 2006) varió entre 39 y $1450 \mathrm{~m}^{3} \mathrm{~s}^{-1}$, con un promedio de $432 \mathrm{~m}^{3} \mathrm{~s}^{-1}$. Las descargas mínimas ocurrieron durante el verano y a principios del otoño (junio-octubre), mientras que otoño, invierno y primavera se 


\section{Results}

Average monthly river discharge for the study period (October 2005 to December 2006) ranged between 39 and $1450 \mathrm{~m}^{3} \mathrm{~s}^{-1}$, with an average of $432 \mathrm{~m}^{3} \mathrm{~s}^{-1}$. Minimum discharge occurred during summer and early fall (June-October), while late fall, winter, and spring were characterized by higher discharges (November-May), with maxima registered on 26 November 2006 corresponding to a flood situation, when hourly river discharge was $7500 \mathrm{~m}^{3} \mathrm{~s}^{-1}$ (fig. 2). The longitudinal salinity structure for low, intermediate, and high inflow of the river during the ebb is presented in figure $3 a-c$.

Averages, ranges, and standard errors for selected variables obtained during this study are presented in table 1 . The concentration of TDC varied from 12.9 to $28.2 \mathrm{mg} \mathrm{L}^{-1}$ and was composed by a higher fraction of DIC (8.4-25.5 $\mathrm{mg} \mathrm{L}^{-1}$ ) and a lower fraction of DOC (1.7-4.5 $\left.\mathrm{mg} \mathrm{L}^{-1}\right)$. In the Douro River estuary, DIC showed a strong conservative behaviour along the salinity gradient (figs. 4, 5a). This pattern of distribution was also observed for TPM and its organic (POM) and inorganic fractions (fig. 5b); i.e., an increasing trend downstream. During low-flow months, the entire estuary was dominated by seawater/brackish water and high DIC and TPM concentrations were found, while lower values of these variables were observed in a higher river flow situation (fig. 5).

In this study the highest values of DOC concentration (November and December 2006; fig. 6b) correspond to high flow and flood situations (fig. 2), causing DOC concentrations to increase sharply within the estuary. The mixing curve generated for all DOC data showed a general non-conservative behaviour of DOC (figs. 7, 8). Independent DOC mixing curves for each month also showed typical deviations from conservative mixing with the exception of January, May, June, and July 2006. During these months a DOC salinity-like gradient was observed, the freshwater end-member being the major DOC source to the estuary (fig. 9a). Nonconservative DOC behaviour was observed in high river inflow situations (November and December 2006) where the entire water column was well mixed, freshwater dominated the entire estuary, and DOC concentrations did not vary along the estuary

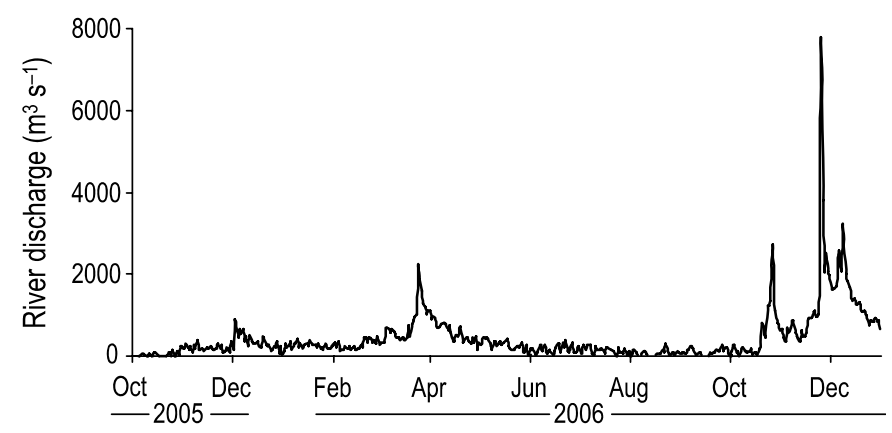

Figure 2. Monthly Douro River discharge during the sampling period. Figura 2. Descarga mensual del Río Duero durante el periodo de muestreo. caracterizaron por altas descargas (noviembre-mayo), con una descarga máxima registrada el 26 de noviembre de 2006 durante el flujo de mareas, cuando la descarga horaria del río alcanzó los $7500 \mathrm{~m}^{3} \mathrm{~s}^{-1}$ (fig. 2). La estructura longitudinal de la salinidad correspondiente a un influjo bajo, intermedio y alto del río durante el reflujo de marea se presenta en la figura 3a-c.

En la tabla 1 se presentan los promedios, intervalos y errores estándar de algunas variables selectas obtenidas durante este estudio. La concentración del CDT varió de 12.9 a $28.2 \mathrm{mg} \mathrm{L}^{-1}$ y estuvo compuesta por una fracción mayor de CID (8.4-25.5 $\mathrm{mg} \mathrm{L}^{-1}$ ) que la de COD (1.7-4.5 $\left.\mathrm{mg} \mathrm{L}^{-1}\right)$. El CID en el estuario del Duero mostró un comportamiento muy conservativo a lo largo del gradiente salino (figs. 4, 5a). Este mismo patrón de distribución también se observó para la MPT y sus fracciones orgánica (MOP) e inorgánica (fig. 5b); es decir, se observó una tendencia a incrementar hacia la boca del estuario, corriente abajo. Durante los meses de flujo bajo todo el estuario estuvo dominado por agua de mar y salobre, y se encontraron concentraciones elevadas de CID y MPT, mientras que en momentos de flujo alto del río se observaron valores menores de estas variables (fig. 5).

En este estudio las mayores concentraciones de COD (noviembre y diciembre de 2006; fig. 6) corresponden a situaciones de alto influjo del río y flujo de mareas (fig. 2), lo que causó que las concentraciones de COD se incrementaran abruptamente dentro del estuario. La curva de mezcla generada
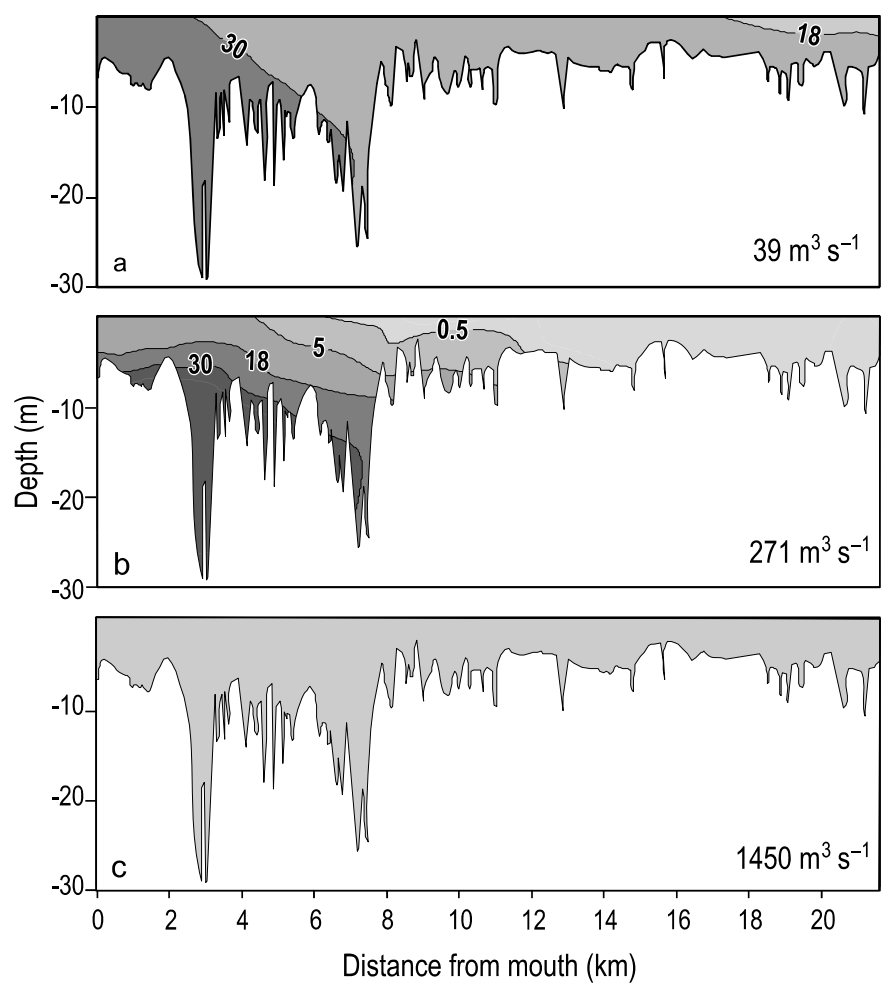

Figure 3. Spatial structure of salinity in the Douro River estuary: (a) October 2005, (b) February 2006, and (c) December 2006 (Salinity < 0.5).

Figura 3. Estructura espacial de la salinidad en el estuario del Duero: (a) octubre de 2005, (b) febrero de 2006, y (c) diciembre de 2006 (Salinidad < 0.5). 
Table 1. Monthly averages ( $\mathrm{mg} \mathrm{L}^{-1}$ ) and standard errors for selected variables obtained in this study (n.a. = not available).

Tabla 1. Promedios mensuales $\left(\mathrm{mg} \mathrm{L}^{-1}\right.$ ) y errores estándar de variables selectas obtenidas en este estudio (n.a. = no disponble).

\begin{tabular}{|c|c|c|c|c|c|c|}
\hline Month/year & TPM & DIC & DOC & DIN & DON & Salinity \\
\hline Oct/05 & $38.8 \pm 2.3$ & $25.0 \pm 0.3$ & $2.3 \pm 0.1$ & $0.2 \pm 0.0$ & $0.5 \pm 0.0$ & $26.5 \pm 1.1$ \\
\hline Nov/05 & $23.1 \pm 2.6$ & $22.0 \pm 0.5$ & $1.7 \pm 0.1$ & $0.2 \pm 0.0$ & $0.6 \pm 0.1$ & $16.0 \pm 2.0$ \\
\hline Dec/05 & $24.8 \pm 3.0$ & $16.9 \pm 0.9$ & $2.2 \pm 0.1$ & $0.3 \pm 0.0$ & $0.9 \pm 0.1$ & $11.0 \pm 2.4$ \\
\hline Jan/06 & $18.6 \pm 2.7$ & $20.3 \pm 0.3$ & $2.0 \pm 0.0$ & $0.4 \pm 0.0$ & $1.0 \pm 0.0$ & $7.8 \pm 1.3$ \\
\hline Feb/06 & $17.5 \pm 2.3$ & $19.7 \pm 0.6$ & $1.7 \pm 0.1$ & $0.4 \pm 0.0$ & $1.1 \pm 0.1$ & $9.7 \pm 2.2$ \\
\hline Mar/06 & $24.2 \pm 3.9$ & $16.2 \pm 0.7$ & $1.6 \pm 0.0$ & $0.3 \pm 0.0$ & $1.1 \pm 0.1$ & $8.7 \pm 1.4$ \\
\hline Apr/06 & $14.0 \pm 2.6$ & $18.5 \pm 0.5$ & $2.0 \pm 0.1$ & $0.5 \pm 0.0$ & $1.2 \pm 0.1$ & $6.1 \pm 1.4$ \\
\hline May/06 & $15.0 \pm 2.0$ & $18.2 \pm 0.5$ & $1.9 \pm 0.0$ & $0.3 \pm 0.0$ & $0.9 \pm 0.0$ & $13.6 \pm 2.6$ \\
\hline Jun/06 & $28.7 \pm 2.7$ & $23.2 \pm 0.4$ & $2.1 \pm 0.1$ & $0.3 \pm 0.0$ & $0.6 \pm 0.1$ & n.a. \\
\hline $\mathrm{Jul} / 06$ & $27.9 \pm 2.8$ & $24.3 \pm 0.4$ & $2.8 \pm 0.1$ & $0.2 \pm 0.0$ & $0.6 \pm 0.0$ & $18.7 \pm 2.3$ \\
\hline Aug/06 & $34.4 \pm 3.0$ & $25.5 \pm 0.4$ & $2.7 \pm 0.1$ & $0.2 \pm 0.0$ & $0.4 \pm 0.0$ & $22.4 \pm 1.9$ \\
\hline Sep/06 & $26.2 \pm 3.0$ & $24.0 \pm 0.4$ & $2.8 \pm 0.1$ & $0.2 \pm 0.0$ & $0.4 \pm 0.0$ & $15.5 \pm 2.3$ \\
\hline Oct/06 & $29.0 \pm 1.3$ & $11.7 \pm 0.1$ & $2.9 \pm 0.0$ & $0.3 \pm 0.0$ & $0.5 \pm 0.0$ & $0.13 \pm 0.0$ \\
\hline Nov/06 & $22.3 \pm 1.6$ & $10.9 \pm 0.6$ & $4.4 \pm 0.1$ & $0.4 \pm 0.0$ & $0.8 \pm 0.0$ & $1.2 \pm 0.2$ \\
\hline Dec/06 & $16.7 \pm 0.4$ & $8.4 \pm 0.7$ & $4.5 \pm 0.2$ & $0.5 \pm 0.0$ & $1.0 \pm 0.1$ & $0.1 \pm 0.0$ \\
\hline
\end{tabular}

(fig. 9c). Under low and intermediate river inflow the nonconservative DOC behavior was related to sewage DOC inputs into the lower and middle estuary (fig. 9b).

A clear seasonality was observed in estuarine DIN and DON concentrations, and both inorganic and organic forms showed the same pattern of variation (fig. 6c-d). Maximum DIN and DON mean concentrations in the estuary occurred when river flow was higher (table 1, fig. 2) and when Douro freshwater occupied almost the entire estuary (fig. 3c). Lower values were registered for decreased river discharge (table 1, fig. 2), when a salt wedge was present in the estuary (fig. 3a). The input of $\mathrm{N}$ to the Douro estuary was dominated by the organic fraction, since the mean DON concentrations (0.4-1.2 $\mathrm{mg} \mathrm{L}^{-1}$ ) were always higher than the mean DIN concentrations $\left(0.2-0.5 \mathrm{mg} \mathrm{L}^{-1}\right)$. Along the estuarine transect, the

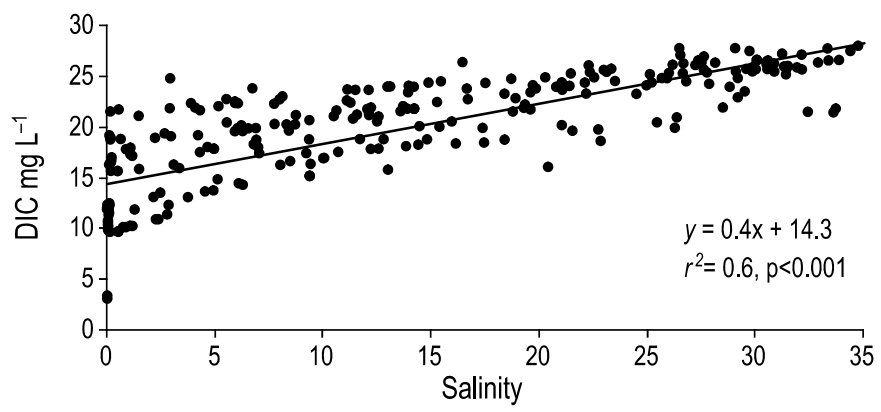

Figure 4. Relationship between dissolved inorganic carbon (DIC) and salinity using all data collected.

Figura 4. Relación entre el carbono inorgánico disuelto (DIC) y la salinidad usando todos los datos recabados. con todos los datos de COD muestra un comportamiento general no conservativo (figs. 7, 8). Las curvas de mezcla independientes para cada mes de COD también mostraron típicamente desviaciones de la mezcla conservativa con excepción de las de enero, mayo, junio y julio de 2006. En esos meses se observó un gradiente del COD similar al de la salinidad, siendo el extremo del agua dulce la mayor fuente de COD para el estuario (fig. 9a). En el COD se observó un comportamiento no
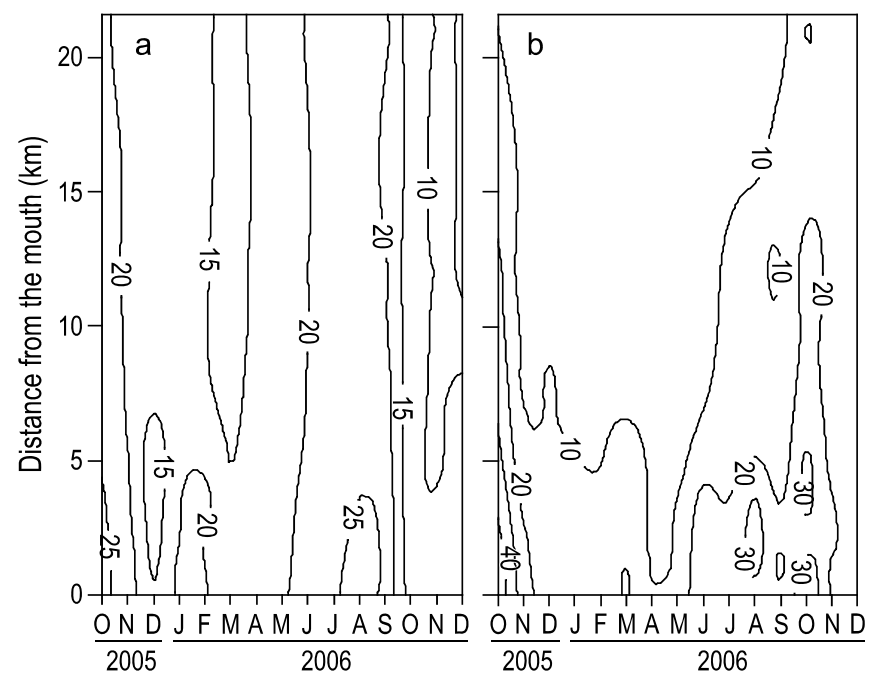

Figure 5. Longitudinal distribution of (a) dissolved inorganic carbon $\left(\mathrm{mg} \mathrm{L}^{-1}\right)$ and (b) total particulate matter ( $\mathrm{mg} \mathrm{L}^{-1}$ ) in the surface water column.

Figura 5. Distribución longitudinal de (a) carbono inorgánico disuelto $\left(\mathrm{mg} \mathrm{L}^{-1}\right)$ y (b) la materia particulada total $\left(\mathrm{mg} \mathrm{L}^{-1}\right)$ en la columna de agua superficial. 

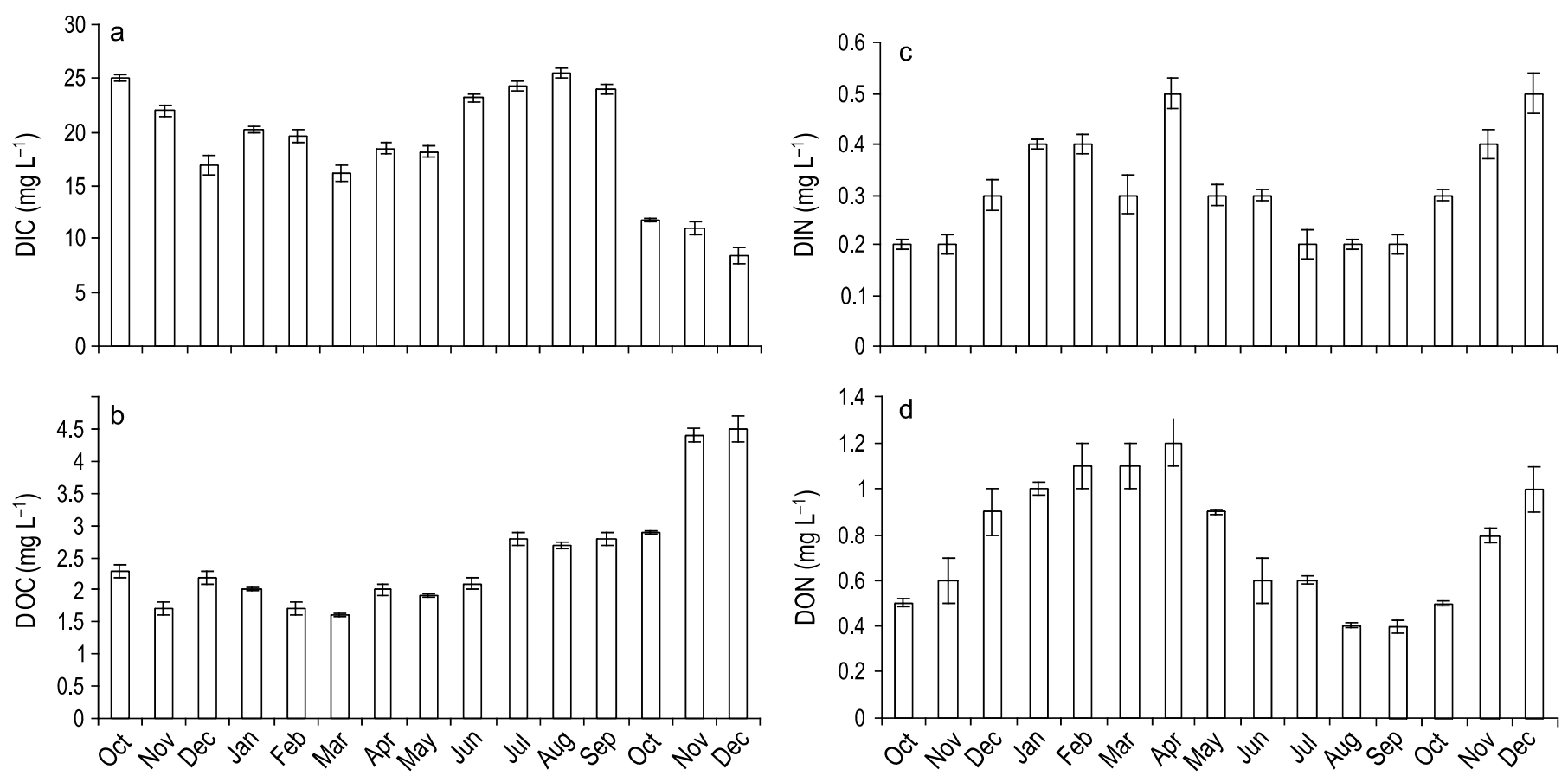

Figure 6. Seasonal variability of (a) dissolved inorganic carbon (DIC), (b) dissolved organic carbon (DOC), (c) dissolved inorganic nitrogen (DIN), and (d) dissolved organic nitrogen (DON). Each value corresponds to monthly averages $\pm \mathrm{SE}$, integrating data from all sampling stations.

Figura 6. Variabilidad estacional de (a) carbono inorgánico disuelto (DIC), (b) carbono orgánico disuelto (DOC), (c) nitrógeno inorgánico disuelto (DIN), y (d) nitrógeno orgánico disuelto (DON). Cada valor corresponde al promedio mensual $\pm \mathrm{EE}$, integrando los datos de todas las estaciones de muestreo.

relationship between DIN and DON and salinity was generally conservative (fig. 10); however, in April, May, and August 2006 a nonconservative behaviour was observed, associated with DON and DIN loads from sewage, since those compounds increased with the increase of fecal coliforms in the middle and lower estuary (an example is given for April 2006 in fig. 11).

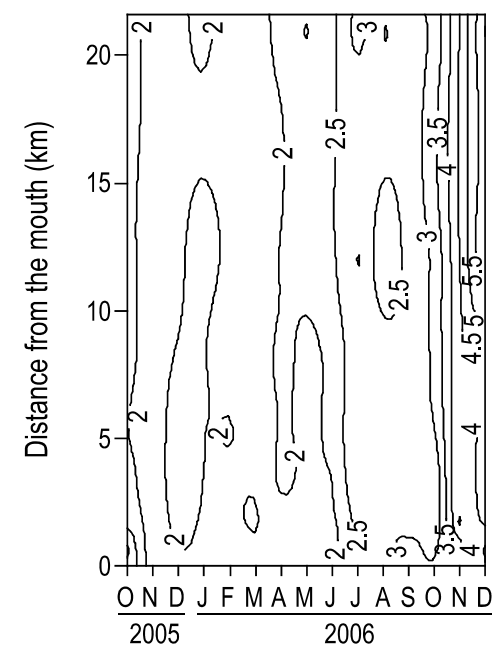

Figure 7. Longitudinal distribution of surface water column dissolved organic carbon $\left(\mathrm{mg} \mathrm{L}^{-1}\right)$ for the monthly survey.

Figura 7. Distribución longitudinal del carbono orgánico disuelto en la columa de agua superficial ( $\mathrm{mg} \mathrm{L}^{-1}$ ) para cada muestreo mensual. conservativo en situaciones de alto influjo fluvial (noviembre y diciembre de 2006) en las que toda la columna de agua estaba bien mezclada, el agua dulce dominaba todo el estuario, y las concentraciones de COD no variaban a lo largo del estuario (fig. 9c). En condiciones de influjo fluvial bajo e intermedio el comportamiento no conservativo del COD se veía relacionado con los aportes de COD de los vertidos de drenaje en la parte baja y media del estuario (fig. 9b).

Se observó una clara estacionalidad en las concentraciones de NID y NOD en el estuario, y ambas formas de nitrógeno mostraron el mismo patrón de variación (fig. 6c, d). Las

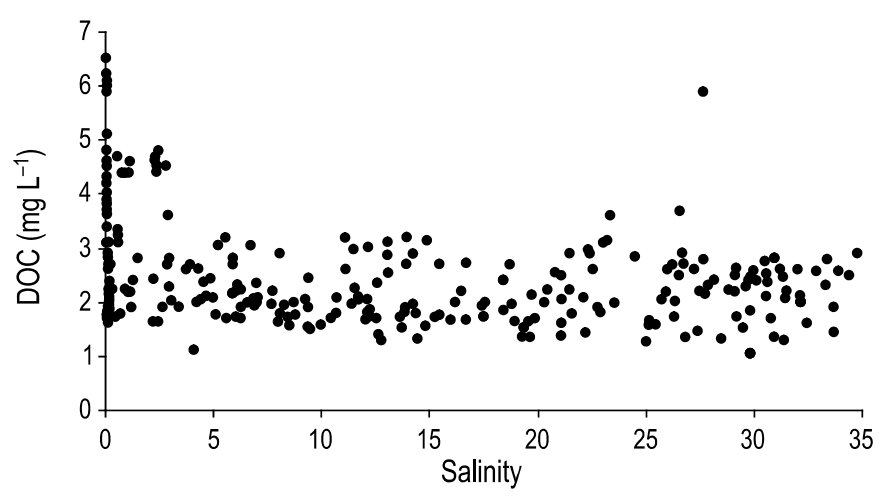

Figure 8. Relationship between dissolved organic carbon (DOC) and salinity using all data collected.

Figura 8. Relación entre carbono orgánico disuelto (DOC) y salinidad usando todos los datos recabados. 


\section{Discussion}

\section{Hydrology}

The primary forcing agent for water circulation in the Douro River estuary was the freshwater inflow. The long-term (1985-2005) Douro River monthly mean discharge was $455 \mathrm{~m}^{3} \mathrm{~s}^{-1}$ (Bordalo and Vieira 2005); however, flow can rise from 0 to $>900 \mathrm{~m}^{3} \mathrm{~s}^{-1}$ in a matter of minutes according to the discharge regime of the upstream dam. Thus, the average monthly river discharge for the study period represents a regular year with regard to river flow.

In the estuary, the position of the salt wedge is a function of the tidal stage and freshwater discharge, controlled by the dam. Though the entire water column was well mixed and freshwater dominated under high river inflow, for discharges $<800 \mathrm{~m}^{3} \mathrm{~s}^{-1}$ threshold, vertical stratification occurs (Vieira and Bordalo 2000). On such occasions, salinity contours showed a salt wedge intruding during the flood, which remained conserved within estuarine boundaries during the ebb.

\section{Dissolved carbon and nitrogen dynamics}

Information on DOC and DON in the Douro River estuary is practically inexistent. Only DOC values based on a single concentraciones medias máximas de NID y NOD ocurrieron cuando el caudal del río era mayor (tabla 1, fig. 2), y cuando el agua dulce del Duero ocupaba casi todo el estuario (fig. 3c), mientras que los valores de estas variables fueron menores al reducirse las descargas del río (tabla 1, fig. 2), coincidiendo con una cuña de agua salada que penetraba en el estuario (fig. 3a). El aporte de $\mathrm{N}$ al estuario del Duero estuvo dominado por la fracción orgánica ya que las concentraciones medias de NOD (0.4-1.2 $\mathrm{mg} \mathrm{L}^{-1}$ ) siempre fueron mayores que las de NID (0.2-0.5 $\left.\mathrm{mg} \mathrm{L}^{-1}\right)$. A lo largo del transecto estuarino la relación entre NID y NOD, y la salinidad, fue generalmente conservativa (fig. 10). Sin embargo, en abril, mayo, y agosto de 2006 se observó un comportamiento no conservativo relacionado con las cargas de NOD y NID del drenaje de aguas negras, debido a que tales compuestos aumentaban con el incremento de las coliformes fecales en las partes media y baja del estuario (en la figura 11 se muestra un ejemplo para abril de 2006).

\section{Discusión}

\section{Hidrología}

El principal forzamiento para la circulación del agua en el estuario del Duero fue el influjo de agua dulce. La descarga media mensual del Río Duero en el largo plazo (1985-2005) es
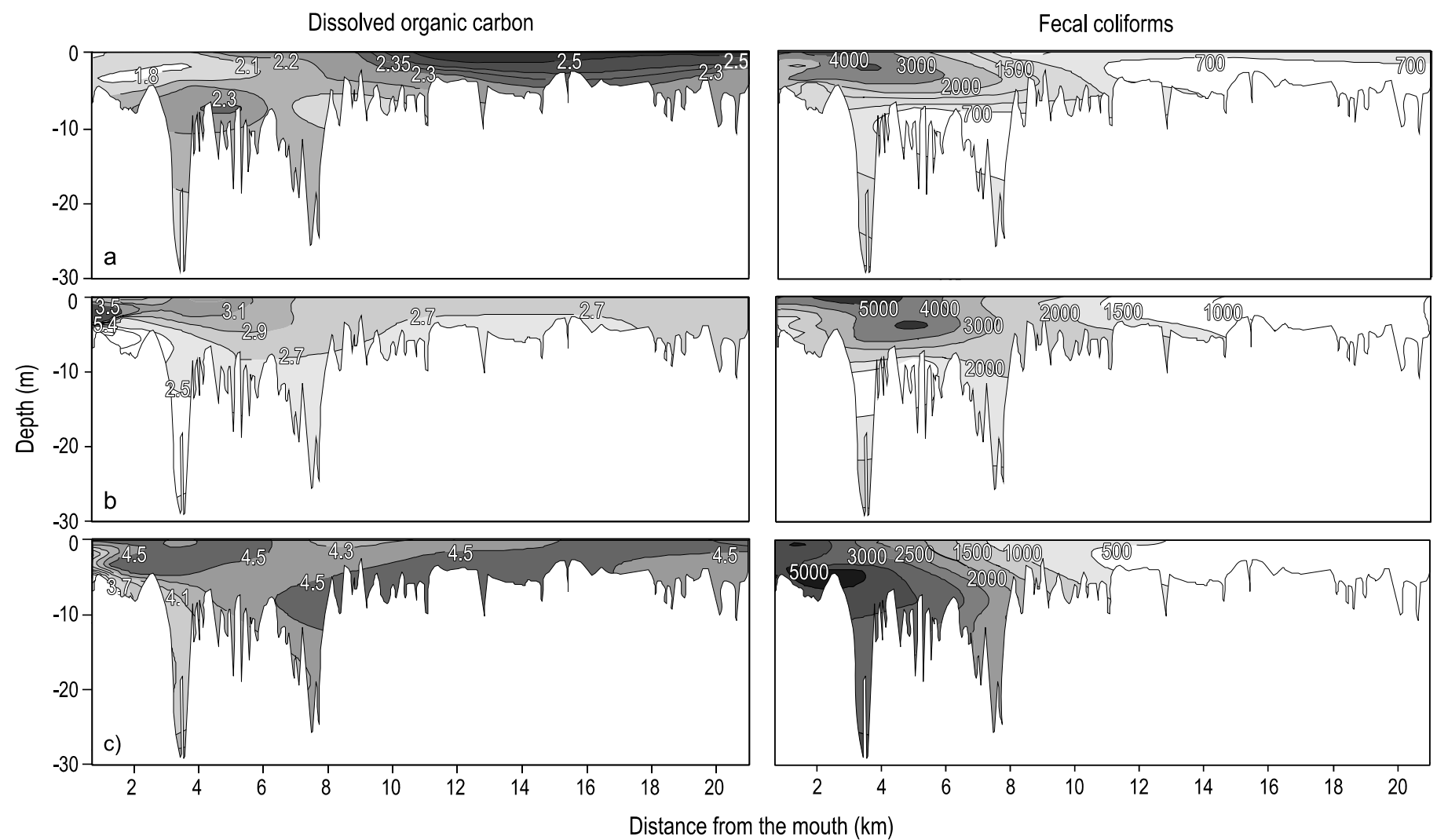

Figure 9. Examples of the spatial distribution of dissolved organic carbon (DOC) concentrations (mg L-1) and fecal coliform counts (CFU $\left.100 \mathrm{~mL}^{-1}\right)$ in the Douro River estuary: (a) June 2006, (b) September 2006, and (c) November 2006.

Figura 9. Ejemplos de la distribución espacial de las concentraciones $\left(\mathrm{mg} \mathrm{L}^{-1}\right)$ de carbono orgánico disuelto (DOC) y los conteos de coliformes fecales (UFC $100 \mathrm{~mL}^{-1}$ ) en el estuario del Duero: (a) junio de 2006, (b) septiembre de 2006 y (c) noviembre de 2006. 
cruise are available in the literature (Abril et al. 2002). Thus, this study is the first comprehensive evaluation of the dissolved organic carbon and nitrogen biogeochemistry in this system. We present monthly measurements made along the salinity gradient, between the river mouth and the upper end of the estuary, in order to understand the estuarine dynamics of those key dissolved organic matter compounds.

In the Douro estuary DIC exhibited a monotonic positive relationship with salinity, indicating an input of DIC into the estuary from coastal waters. This pattern of distribution along the salinity gradient was also observed for TPM. The dynamics of TPM in estuaries depends on sediment transport from rivers and on locally derived suspended sediments, associated with a turbidity maximum or estuarine bed sediment resuspension (Uncles et al. 2000). The dynamics of TPM in the estuary may be explained by the high level of sediment retention by the upstream network of over 50 large dams that removes suspended matter from the water discharged into the estuary, but also by the sewage disposal in the middle and lower estuary and higher resuspension rates due to greater current velocities in the lower section of the estuary (Azevedo et al. 2006, 2008). Nevertheless, TPM loads to the estuary are relatively low compared with other European estuarine systems, such as the Sado, Gironde, and Scheldt estuaries (Cabeçadas et al. 1999).

In this study, DOC concentrations are in the lower range compared with other estuarine systems (c.f., Abril et al. 2002, Goni et al. 2003, Harrison et al. 2005). As DOC is a major component of the riverine organic matter imported to estuaries (Spitz and Ittekkot 1991), it usually decreases linearly with increasing salinity; however, this does not necessarily imply a
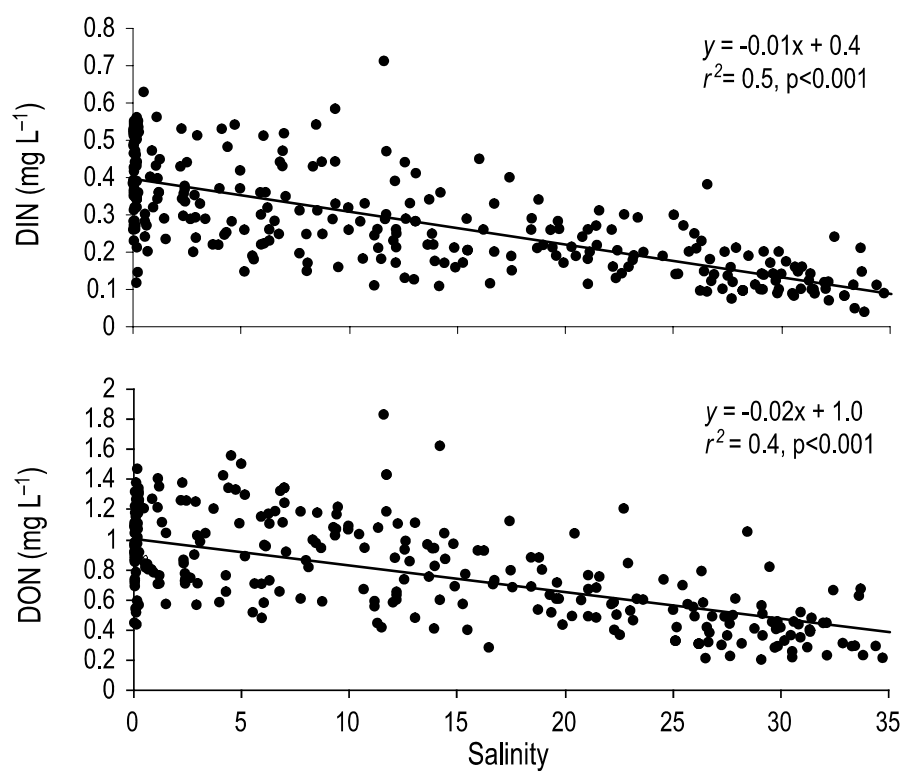

Figure 10. Relationship between dissolved inorganic nitrogen (DIN) and dissolved organic nitrogen (DON) with salinity using all data collected.

Figura 10. Relación entre nitrógeno inorgánico disuelto (DIN) y nitrógeno orgánico disuelto (DON) con la salinidad usando todos los datos recabados.
$455 \mathrm{~m}^{3} \mathrm{~s}^{-1}$ (Bordalo y Vieira 2005), no obstante el flujo puede aumentar de 0 a $>900 \mathrm{~m}^{3} \mathrm{~s}^{-1}$ en cuestión de minutos, según el régimen de descargas de la presa río arriba. Por ello, el promedio mensual de descarga fluvial del periodo de estudio representa un año regular en lo que concierne al flujo del río.

En el estuario del Duero la cuña de agua salada se ubica en función del estado de la marea y la descarga de agua dulce controlada por la presa. Aunque bajo un alto influjo fluvial toda la columna de agua se encontró bien mezclada y dominada por el agua dulce, con descargas por debajo del umbral de $800 \mathrm{~m}^{3} \mathrm{~s}^{-1}$ se presenta estratificación vertical (Vieira y Bordalo 2000). En este último caso los contornos de salinidad mostraron una cuña de agua salada penetrando durante el flujo de marea, misma que se conservaba dentro de los límites del estero durante el reflujo.

\section{Dinámica del carbono y nitrógeno disueltos}

No existe información acerca del COD y el NOD en el estuario del Duero. En la literatura sólo se encuentran disponibles valores de COD basados en un solo crucero (Abril et al. 2002), por lo que este estudio constituye la primera evaluación comprehensiva de la biogeoquímica de estos grupos de compuestos en el sistema. Aquí se presentan mediciones mensuales hechas a lo largo del gradiente salino entre la boca del río y el

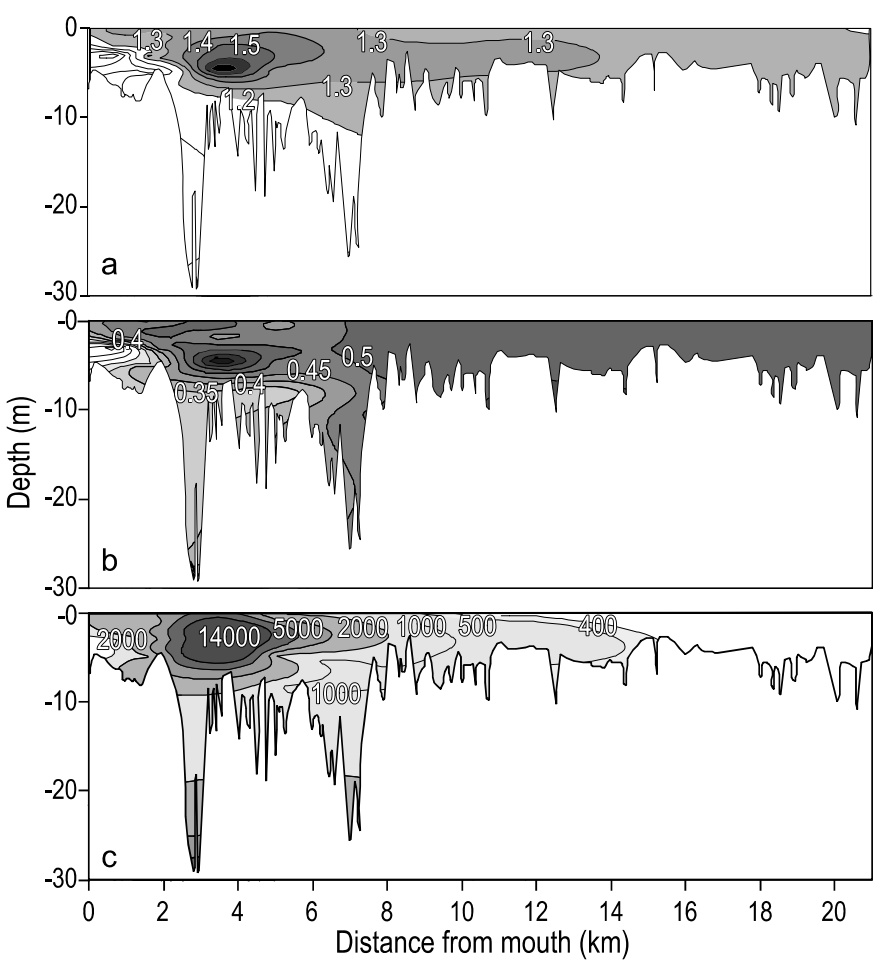

Figure 11. Longitudinal spatial distribution of (a) dissolved organic nitrogen and (b) dissolved inorganic nitrogen concentrations ( $\left.\mathrm{mg} \mathrm{L}^{-1}\right)$, and (c) fecal coliform counts (CFU $100 \mathrm{~mL}^{-1}$ ) for April 2006 in the Douro River estuary.

Figura 11. Distribución espacial longitudinal de las concentraciones (mg $\mathrm{L}^{-1}$ ) de (a) nitrógeno orgánico disuelto y (b) conteos de coliformes fecales (UFC $100 \mathrm{~L}^{-1}$ ) en abril de 2006 en el estuario del Duero. 
conservative behaviour since it could be due to the existence of simultaneous sources and sinks resulting in almost null net changes (Moran et al. 1999, Raymond and Bauer 2000, Abril et al. 2002, Goni et al. 2003). The general nonconservative behaviour of DOC observed in the Douro estuary suggests the occurrence of DOC sources that could differ in time and space depending on external (river discharge, pulses of sewage discharge) and/or internal inputs. The results demonstrated sewage DOC inputs into the lower and middle estuary, since a good overlap between fecal coliform counts (an indicator of sewage discharges) and DOC concentrations was observed (fig. 9c). The human-induced contamination of the lower and middle Douro estuary has been described (Bordalo 2003), and was previously found to enhance respiration, contributing to the heterotrophy situation observed all year round in the pelagic metabolism in the lower and middle stretches of the estuary (Azevedo et al. 2006).

Most of the studies on estuarine systems have focused on inorganic nitrogen (DIN, nitrate + nitrite + ammonium) biogeochemistry. The information on DON is scarcer, even though it represents an important pool of reduced N, since a considerable portion of the $\mathrm{N}$ inputs to coastal ecosystems is in the form of organic N (e.g., Meybeck 1982, Seitzinger and Sanders 1997, Lewis et al. 1999, Seitzinger et al. 2002). In this study, both inorganic and organic forms showed the same pattern of variation, denoting a common source and behaviour. A major fluvial influence on Douro estuarine water DIN and DON concentrations was observed. The input of $\mathrm{N}$ to the Douro estuary was dominated by the organic fraction; on average, DON concentrations represented $52-88 \%$ of the TDN. While this is not always the case (Mortazavi et al. 2000), DON is frequently more abundant than DIN in estuarine systems (Eyre and Pont 2003), particularly in systems that receive untreated sewage, due to the time required for the biological transformation of the organic forms. Anthropogenic DON and DIN loads into the Douro estuary were not as evident as observed for DOC, since the conservative behaviour of these compounds dominated in most of the surveys. However, in April, May, and August 2006, DON and DIN loads from sewage were registered (fig. 11). As $\mathrm{N}$ is the primary nutrient-limiting resource for algal and microbial production in many marine and some freshwater environments, increases in DON inputs can markedly alter those ecosystems (e.g., Ryther and Dunstan 1971, Vitousek and Howarth 1991). Thus, studying the effects of human-induced contamination of DON loading on phytoplankton production, biomass, and species composition is of considerable interest in future research, particularly in the Douro estuary where the anthropogenic pressure is evident.

\section{Annual dissolved carbon and nitrogen balance}

The magnitude and seasonality of freshwater input to the Douro estuary is mainly determined by the Douro River flow pattern, managed according to hydroelectric production requirements rather than environmental needs (Azevedo et al. extremo superior del estuario, esto a fin de entender la dinámica estuarina de los componentes claves de la materia orgánica disuelta.

El CID en el estuario del Duero mostró una relación monotónica positiva con la salinidad, lo que indica un aporte de CID al estuario desde las aguas costeras adyacentes. Este patrón de distribución a lo largo del gradiente salino también se observó para la MPT, cuya dinámica en los estuarios depende del transporte de sedimentos de los ríos y de sedimentos suspendidos a la deriva localmente, asociados a un máximo de turbidez o a la resuspensión de sedimentos del lecho marino (Uncles et al. 2000). La dinámica de la MPT en el estuario del Duero puede ser explicada por el alto nivel de retención de sedimentos por la red de más de 50 grandes presas río arriba que retienen la materia suspendida del agua descargada hacia el estuario, pero también por el vertido de aguas negras en las partes media y baja del estuario y por las mayores tasas de resuspensión de sedimentos causadas por las mayores velocidades de la corriente en la parte baja del mismo (Azevedo et al. 2006, Azevedo et al. 2008). No obstante, las cargas de MPT al estuario del Duero son relativamente bajas en comparación con otros sistemas estuarinos europeos tales como los estuarios del Sado, la Gironda y Scheldt (Cabeçadas et al. 1999).

En este estudio las concentraciones de COD se encontraron en un rango menor en comparación con otros sistemas estuarinos (c.f. Abril et al. 2002, Goni et al. 2003, Harrison et al. 2005). Dado que el COD es un componente mayor de la materia orgánica fluvial importada hacia los estuarios (Spitzy e Ittekkot 1991) usualmente disminuye linealmente conforme se incrementa la salinidad. Sin embargo, esto no necesariamente implica un comportamiento conservativo ya que esto podría deberse a la existencia de fuentes y sumideros simultáneos que resultarían en cambios netos casi nulos (Moran et al. 1999, Raymond y Bauer 2000, Abril et al. 2002, Goni et al. 2003). El comportamiento general no conservativo del COD observado en el estuario del Duero sugiere la existencia de fuentes que podrían variar temporal y espacialmente dependiendo de los aportes externos (descarga fluvial, pulsos de descarga de aguas negras) y o internos. Los resultados mostraron aportes de COD de drenaje en las partes baja y media del estuario que coincidieron en buena medida con los conteos de coliformes fecales (indicadoras de descargas de aguas negras) y con las concentraciones de COD observadas (fig. 9c). La contaminación inducida por el hombre en las partes baja y media del estuario del Duero ya había sido descrita por Bordalo (2003), y previamente se había encontrado que incrementaba la respiración del sistema contribuyendo a la heterotrofia observada todo el año en el meta-bolismo pelágico de las porciones baja y media del estuario del Duero (Azevedo et al. 2006).

La mayoría de los estudios en sistemas estuarinos están enfocados en la bioquímica del nitrógeno inorgánico (NID, nitrato + nitrito + amonio), mientras que los datos sobre NOD son más bien escasos. Sin embargo, éste representa una reserva importante de $\mathrm{N}$ reducido, ya que una porción considerable de los aportes de $\mathrm{N}$ a los ecosistemas costeros es en forma 
2008). In this study, DIN and DON exported from the estuary were estimated from mean water column concentrations and river discharge. Annually integrated DON calculations resulted in an export to the coastal zone of $1.2 \times 10^{7} \mathrm{~kg} \mathrm{yr}^{-1}$ of DON. This corresponds to $125 \mathrm{~kg} \mathrm{yr}^{-1}$ of DON that is exported per square kilometer of Douro basin area $\left(98,000 \mathrm{~km}^{2}\right)$. These values differ from the predicted values of DON exported to coastal waters by worldwide individual basins calculated by the global DON model developed by Harrison et al. (2005), which yielded lower values for the Douro River watershed (30-50 $\mathrm{kg} \mathrm{N} \mathrm{km}^{-2} \mathrm{yr}^{-1}$ ). In the case of DOC, although the Douro River represents its major source, this study revealed that additional anthropogenic sources were present in the middle and lower estuary. The annual DOC export to the coastal zone was estimated at $4.2 \times 10^{7} \mathrm{~kg} \mathrm{yr}^{-1}$ or $431 \mathrm{~kg} \mathrm{C} \mathrm{km}^{-2} \mathrm{yr}^{-1}$, when the Douro River watershed is considered. In contrast to DON, the Douro estuary DOC exports calculated in this study were similar to the higher range of values predicted by global river DOC export models (Harrison et al. 2005), which forecasted $170-340 \mathrm{~kg} \mathrm{C} \mathrm{km}^{-2} \mathrm{yr}^{-1}$ for the geographic area of the Douro watershed .

\section{Acknowledegments}

We thank L Lima for laboratory assistance. This work was partially funded by the Porto Water and Wastewater Board (SMAS), through a contract awarded to AAB.

\section{References}

APHA, WWA, WEF. 1992. Standard Methods for the Examination of Water and Wastewater. 18th ed. American Public Health Association, Washington, DC, pp. 256-257.

Abril G, Nogueira M, Etcheber H, Cabeçadas G, Lemaire E, Brogueira MJ. 2002. Behaviour of organic carbon in nine contrasting European estuaries. Estuar. Coast. Shelf Sci. 54: 241-262.

Azevedo IC, Duarte PM, Bordalo AA. 2006. Pelagic metabolism of the Douro estuary (Portugal): Factors controlling primary production. Estuar. Coast. Shelf Sci. 69: 133-146.

Azevedo IC, Duarte PM, Bordalo AA. 2008. Understanding spatial and temporal dynamics of key environmental characteristics in a mesotidal Atlantic estuary (Douro, NW Portugal). Estuar. Coast. Shelf Sci. 76: 620-633.

Bordalo A. 2003. Microbiological water quality in urban coastal beaches: The influence of water dynamics and optimization of the sampling strategy. Water Res. 37: 3233-3241.

Bordalo A, Vieira M. 2005. Spatial variability of phytoplankton, bacteria and viruses in the mesotidal salt wedge Douro Estuary (Portugal). Estuar. Coast. Shelf Sci. 63: 143-154.

Cabeçadas G, Nogueira M, Brogueira MJ. 1999. Nutrient dynamics and productivity in three European estuaries. Mar. Pollut. Bull. 38: 1092-1096.

Eyre BD, Pont D. 2003. Intra- and inter-annual variability in the different forms of diffuse nitrogen and phosphorus delivered to seven sub-tropical east Australian estuaries. Estuar. Coast. Shelf Sci. 57: 137-148.

Findlay SEG, Sinsabaugh RL. 2003. Aquatic Ecosystems: Interactivity of Dissolved Organic Matter. Elsevier, New York, 512 pp. orgánica (e.g. Meybeck 1982, Seitzinger y Sanders 1997, Lewis et al. 1999, Seitzinger et al. 2002). En este estudio tanto la forma inorgánica como la orgánica mostraron el mismo patrón de variación, lo que denota una fuente y un comportamiento común. Se observó una influencia fluvial preponderante en las concentraciones de NID y NOD en las aguas del estuario del Duero. Los aportes de $\mathrm{N}$ al estuario del Duero estuvieron dominados por la fracción orgánica; en promedio, las concentraciones de NOD representaron de $52 \%$ a $88 \%$ del NDT. Mientras que éste no es siempre el caso (Mortazavi et al. 2000), el NOD es frecuentemente más abundante que el NID en los sistemas estuarinos (Eyre y Pont 2003), particularmente en sistemas que reciben aguas negras no tratadas, debido al tiempo necesario para la transformación biológica de las formas orgánicas. Las cargas antropogénicas de NOD y NID al estuario del Duero no fueron tan evidentes como las observadas para el COD, ya que el comportamiento conservativo de estos compuestos dominó en la mayoría de los muestreos. Sin embargo, en abril, mayo y agosto de 2006 se registraron cargas de NOD y NID provenientes de aguas negras (fig. 11). Dado que el $\mathrm{N}$ es el recurso limitante de nutrientes primordial para la producción algal y microbiana en muchos ambientes marinos y algunos de agua dulce, los aumentos en los aportes de NOD pueden alterar notablemente tales ecosistemas (e.g., Ryther y Dunstan 1971, Vitousek y Howarth 1991). Por ello resulta de considerable interés investigar los efectos de la contaminación inducida por el hombre en forma de sobrecargas de NOD a la producción, biomasa y composición específica del fitoplancton, particularmente en el estuario del Duero donde la presión antropogénica es evidente.

\section{Balance anual de carbono y nitrógeno disuelto}

La magnitud y estacionalidad del aporte de agua dulce al estuario del Duero se encuentra determinada principalmente por el patrón de flujo del Río Duero, el cual es manejado más de acuerdo con las necesidades de producción hidroeléctrica que con criterios ambientales (Azevedo et al. 2008). En este estudio se estimaron el NID y NOD exportados por el estuario del Duero a partir de sus concentraciones medias en la columna de agua y las descargas fluviales. Los cálculos integrados anualmente resultaron en una exportación hacia la zona costera de $1.2 \times 10^{7} \mathrm{~kg}$ año-1 de NOD, lo que corresponde a $125 \mathrm{~kg}$ año ${ }^{-1}$ de NOD exportado por kilómetro cuadrado de la cuenca del Duero $\left(98,000 \mathrm{~km}^{2}\right)$. Estos valores difieren de los predichos para el NOD exportado hacia aguas costeras por cuencas individuales alrededor del mundo calculados por el modelo global desarrollado por Harrison et al. (2005) para el NOD, el cual arrojó valores menores para la cuenca hidrológica del Río Duero (30-50 kg N km-2 año-1). En el caso del COD, aunque el río representa su fuente principal, este estudio reveló que existen fuentes antropogénicas adicionales en las partes media y baja del estuario. Al considerar la cuenca hidrológica del Duero, la exportación anual de COD hacia la zona costera se 
Goldman JC, Caron DA, Dennett MR. 1987. Regulation of gross growth efficiency and ammonium regeneration in bacteria by substrate C:N ratio. Limnol. Oceanogr. 32: 1239-1252.

Goni MA, Teixeira MJ, Perkey DW. 2003. Sources and distribution of organic matter in a river-dominated estuary (Winyah Bay, SC, USA). Estuar. Coast. Shelf Sci. 57: 1023-1048.

Grasshoff K, Ehrhardt M, Kremling K. 1983. Methods of Seawater Analysis. Verlag Chemie, Weinheim.

Harrison JA, Nina C, Seitzinger SP. 2005. Global patterns and sources of dissolved organic matter export to the coastal zone: Results from a spatially explicit, global model. Global Biogeochem. Cycles 19: 1-16.

Hopkinson C, Vallino J. 1995. The relationships among Man’s activities in watersheds and estuaries: A model of runoff effects on patterns of estuarine community metabolism. Estuaries 18: 598-621.

ISO. 1990. Water Quality: Detection and Enumeration of Coliform Bacteria, Thermotolerant Coliform Bacteria and Escherichia coli, ISO 9308/1. International Organization for Standardization, Switzerland.

Jones MN. 1984. Nitrate reduction by shaking with cadmium: Alternative to cadmium columns. Water Res. 18: 643-646.

Lewis WM, Melack JM, Mcdowell WH, Mcclain M, Richey JE. 1999. Nitrogen yields from undisturbed watersheds in the Americas. Biogeochemistry 46:149-162.

Ludwig W, Probst JL, Kempe S. 1996. Predicting the oceanic input of organic carbon by continental erosion. Global Biogeochem. Cycles 10: 23-41.

Meybeck M. 1982. Carbon, nitrogen, and phosphorus transport by world rivers. Am. J. Sci. 282: 401-450.

Moran MA, Sheldon WM, Sheldon JE. 1999. Biodegradation of riverine dissolved organic carbon in five estuaries of the southeastern United States. Estuaries 22: 55-64.

Mortazavi B, Iverson R, Huang W, Lewis G, Caffrey JM. 2000. Nitrogen budget of Apalachicola Bay, a bar-built estuary in the northeastern Gulf of Mexico. Mar. Ecol. Prog. Ser. 195: 1-14.

Nagata T. 2000. Production mechanisms of dissolved organic matter. In: Kirchman DL (ed.), Microbial Ecology of the Oceans. WileyLiss, New York, pp. 121-152.

Nixon SW, Ammerman JW, Atkinson LP, Berounsky VM, Billen G, Boicourt WC, Boynton WR, Church TM, DiToro DM, Elmgren R, Garber JH, Giblin AE, Jahnke RA, Owens NJP, Pilson MEQ, Seitzinger SP. 1996. The fate of nitrogen and phosphorus at the land-sea margin of the North Atlantic Ocean. Biogeochemistry 35: $141-180$. estimó en $4.2 \times 10^{7} \mathrm{~kg}_{\text {año }}{ }^{-1}$ ó $431 \mathrm{~kg} \mathrm{C} \mathrm{km}^{-2}$ año- $^{-1}$. En contraste con lo que sucede con el NOD, la exportación de COD por el estuario del Duero calculada en este estudio resultó similar al mayor intervalo de valores predichos por los modelos globales de exportación de COD de los ríos (Harrison et al. 2005), cuyas predicciones para el área geográfica del Duero fueron de $170-340 \mathrm{~kg} \mathrm{C} \mathrm{km}^{-2}$ año ${ }^{-1}$.

\section{Agradecimientos}

Agradecemos a L Lima su asistencia en el laboratorio. Este trabajo fue parcialmente financiado por Serviços Municipalizados de Águas e Saneamento do Porto (SMAS) mediante un contrato asignado a AA Bordalo.

Traducido al español por Manuel Gardea-Ojeda.

Raymond PA, Bauer JE. 2000. Bacteria consumption of DOC during transport through a temperate estuary. Aquat. Microb. Ecol. 22: 1-2.

Ryther JH, Dunstan WM. 1971. Nitrogen, phosphorus and eutrophication in the coastal marine environment. Science 171: 1008-1013.

Seitzinger SP, Sanders RW. 1997. Contribution of dissolved organic nitrogen from rivers to estuarine eutrophication. Mar. Ecol. Prog. Ser. 159: 1-12.

Seitzinger SP, Sanders RW, Styles R. 2002. Bioavailability of DON from natural and anthropogenic sources to estuarine plankton. Limnol. Oceanogr. 47: 353-366.

Spitz A, Ittekkot V. 1991. Dissolved and particulate organic matter in rivers. In: Mantora RFC, Martin JM, Wallast R (eds.), Ocean Margin Processes in Global Change. Wiley, pp. 5-16.

Uncles RJ, Bloomer NJ, Frickers PE, Griffiths ML, Harris C, Howland RJM, Morris AW, Plummer DH, Tappin AD 2000. Seasonal variability, temperature, turbidity and suspended chlorophyll in the Tweed Estuary. Sci. Total Environ. 251: 115-124.

Vieira MEC, Bordalo AA. 2000. The Douro Estuary (Portugal): A mesotidal salt wedge. Oceanol. Acta 23: 585-594.

Vitousek PM, Howarth RW. 1991. Nitrogen limitation on land and in the sea: How can it occur? Biogeochemistry 13: 87-115.

Zar JH. 1996. Biostatistical Analysis. Prentice-Hall, Englewood Cliffs, New Jersey.

Recibido en febrero de 2008; aceptado en agosto de 2008. 